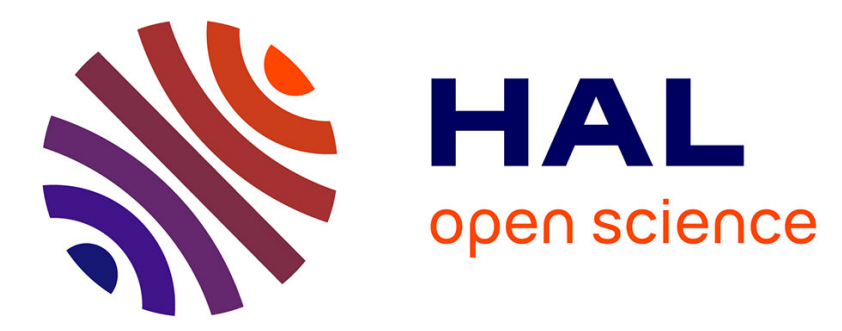

\title{
L'ARN satellite du virus de la mosaïque du concombre. I. - Comparaison de l'aptitude à induire la nécrose de la tomate d'ARN satellites isolés de plusieurs souches du virus
}

Mireille Jacquemond, Hervé Lot

\section{To cite this version:}

Mireille Jacquemond, Hervé Lot. L'ARN satellite du virus de la mosaïque du concombre. I. - Comparaison de l'aptitude à induire la nécrose de la tomate d'ARN satellites isolés de plusieurs souches du virus. Agronomie, 1981, 1 (10), pp.927-932. 10.1051/agro:19811015 . hal-02726970

\section{HAL Id: hal-02726970 \\ https: / hal.inrae.fr/hal-02726970}

Submitted on 2 Jun 2020

HAL is a multi-disciplinary open access archive for the deposit and dissemination of scientific research documents, whether they are published or not. The documents may come from teaching and research institutions in France or abroad, or from public or private research centers.
L'archive ouverte pluridisciplinaire HAL, est destinée au dépôt et à la diffusion de documents scientifiques de niveau recherche, publiés ou non, émanant des établissements d'enseignement et de recherche français ou étrangers, des laboratoires publics ou privés. 


\section{L'ARN satellite du virus de la mosaïque du concombre I. - Comparaison de l'aptitude à induire la nécrose de la tomate d'ARN satellites isolés de plusieurs souches du virus}

Mireille JACQUEMOND \& Hervé LOT

I.N.R.A., Station de Pathologie végétale, Centre de Recherches agronomiques d'Avignon, F 84140 Montfavet.

\section{RÉSUMÉ}

Virus de la mosaïque du concombre,

ARN satellite,

Tomate,

Nécrose,

Réduction de symptômes.
Des tomates infectées par le VMC extériorisent des symptômes de mosaïque et filiformisme ou de nécrose létale. Le développement du syndrome nécrotique est caractéristique de la présence d'ARN-5 (satellite du VMC) dans les nucléoprotéines. De plus, l'addition d'ARN-5 purifié à partir de différentes souches du virus $\left(\mathrm{I}_{17} \mathrm{~N}, \mathrm{D}, \mathrm{TL}, \mathrm{S}\right)$ à des isolats dépourvus d'ARN satellite et inducteurs de mosaïque et filiformisme $\left(\mathrm{I}_{17} \mathrm{~F}, \mathrm{~K}\right.$, $\mathrm{M})$ provoque le développement de la nécrose.

Une souche particulière de VMC (la souche R), pourvue d'ARN-5, n'induit pas de nécrose chez les tomates qu'elie infecte. L'adjonction de cet ARN satellite aux génomes viraux $I_{17} F, K$ ou $M$ se traduit par la réduction des symptômes de filiformisme résultant de l'infection par le seul génome viral.

Ces résultats suggèrent l'existence de deux types d'ARN satellite se différenciant par leur aptitude à induire ou non la nécrose létale de la tomate. Lorsqu'ils sont simultanément présents dans l'inoculum, l'effet provoqué par la synthèse de l'ARN satellite nécrogène domine si sa concentration initiale est suffisante pour que toutes les tomates où il a été inoculé seul avec le génome viral se nécrosent.

\section{SUMMARY \\ Cucumber mosaic virus, Satellite RNA, Tomato, \\ Necrosis, \\ Attenuation of symptoms}

\begin{abstract}
Cucumber mosaic virus-associated RNA-5 - I. - Comparison of the aptitude to induce tomato necrosis of satellite RNA isolated from various CMV strains

Tomato plants when infected with cucumber mosaic virus may develop mosaic and fern leaf symptoms or lethal necrosis. The necrotic syndrome is associated with the presence of RNA-5 (CMV-satellite) in the nucleoproteins. When added to different viral genomes $\left(I_{17} F, K, M\right)$ inducing fern leaf symptoms, RNA-5 of various strains $\left(I_{17} N, D, T L, S\right)$ provokes the development of typical necrosis on so infected tomato plants. A particular CMV strain ( $R$ strain) cannot induce the necrosis, although great amount of RNA-5 is synthesized on tomato. The inoculation of the genomes $I_{17} F, K$ or M with CMV-R satellite RNA results in an important decrease of the symptoms of mosaic and fern leaf caused by the infection with the genome alone. These results suggest that there are two types of satellite RNAs differing by their capacity to induce the tomato necrosis. When they are present together in the inoculum, the plants essentially develop necrosis if the concentration of the "necrogen "satellite RNA is important enough to induce necrosis in all the tomato plants where it has been inoculated with the genome RNAs. In other cases, it seems that the two satellite RNAs may interfere.
\end{abstract}

\section{INTRODUCTION}

L'ARN du virus de la mosaïque du concombre (VMC) est constitué de trois $\mathrm{ARN}$ génomiques (ARN-1, 2 et 3) (PEDEN \& SYMONS, 1973; LOT et al., 1974) et d'un ARN subgénomique (ARN-4) numérotés par ordre de poids moléculaire décroissant. Certaines préparations du virus sont pourvues d'un $5^{\mathrm{c}}$ composant de poids moléculaire $0,11 \times 10^{6}$ Daltons, dénommé ARN-5 (KAPER et al., 1976; TAKANAMI et al., 1977) puis CARNA-5 (KAPER
\& TOUSIGNANT, 1977), ou SAT-RNA (GOULD et al. 1978). Encapsidé dans la nucléoprotéine virale (LOT \& KAPER, 1976), il est totalement dépendant, pour sa réplication des ARN du génome. L'absence de larges homologies, de séquence entre cet ARN surnuméraire et les ARN du VMC confirme qu'il s'agit d'un satellite (DIAZ-RuIZ \& KAPER, 1977 ; LOT et al., 1977 ; GOULD et al., 1978).

Chez la tomate (Lycopersicon esculentum Mill.), le VMC induit 2 types de symptômes : mosaique et/ou filiformisme ou nécrose létale. La maladie nécrotique, apparue vers 1970 
TABLEAU 1

Origine et groupe d'appartenance des souches de VMC utilisées

Origin and group of CMV strains

\begin{tabular}{|c|c|c|c|}
\hline Souche & Origine de l'isolat & Groupc & Obtentcur \\
\hline $\mathrm{I}_{17} \mathrm{~F}$ & Lycopersicon esculentum Mill. (France) & $\mathrm{C}$ & QUIOT \& LATERROT, 1975 \\
\hline $\mathrm{I}_{17} \mathrm{~N}$ & Lycopersicon esculentum Mill. (France) & $\mathrm{C}$ & QUIOT \& LATERROT, 1975 \\
\hline $\mathrm{D}$ & Lycopersicon esculentum Mill. (France) & $\mathrm{C}$ & MARROU, 1967 \\
\hline TL & Stellaria media L. (France) & $\mathrm{C}$ & Quiot, 1972 \\
\hline $\mathbf{M}$ & Vinca rosea L. (Maroc) & $\mathrm{C}$ & BOVÉ, 1977 \\
\hline $\mathbf{S}$ & Lupinus sp. (Afrique du Sud) & B & VAN REGENMORTEL, 1961 \\
\hline $\mathbf{K}$ & Vinca rosea L. (Maroc) & B & BOVÉ, 1977 \\
\hline $\mathbf{R}$ & Ranunculus asiaticus L. (France) & B & DEVERGNE et al., 1969 \\
\hline
\end{tabular}

en France, avait été attribuée à certains isolats de VMC (Marrou et al., 1973; Putz et al., 1974). Plus tard, KAPER \& WATERWORTH (1977) ont montré que l'association de l'ARN-5 ou CARNA-5 au génome viral provoque chez la tomate, le développement d'un syndrome nécrotique comparable à celui qui avait été décrit en France par les divers auteurs.

Les résultats que nous présentons ici confirment la responsabilité de l'ARN-5 associé au VMC dans l'induction du syndrome nécrotique. Nous démontrons toutefois que l'ARN-5 isolé d'une souche particulière est par contre incapable de modifier le type de symptômes conférés par les seuls ARN génomiques du virus inducteur de mosaïque filiforme et suggérons l'existence en France de 2 types d'ARN satellites.

\section{MATÉRIEL ET MÉTHODES}

\section{A. Les souches de virus. Méthode d'inoculation}

Les souches de virus utilisées (tabl. 1) ont été caractérisées selon les critères de symptomatologie, sérologie et sensibilité thermique qui ont permis de définir 2 groupes de souches (MARCHOUX, 1975). Elles proviennent du clonage d'isolats naturels par transferts successifs d'une lésion locale sur Vigna sinensis (L.) Endl. Les souches $\mathrm{I}_{17} \mathrm{~F}$ et $\mathrm{I}_{17} \mathrm{~N}$ sont issues du clonage d'un même isolat naturel.

Dans tous les cas, la réaction d'une large gamme d'hôtes et l'observation au microscope électronique des suspensions virales purifiées attestaient que ces souches n'étaient pas contaminées par d'autres virus.

Les tomates, var. "Monalbo ", sont inoculées à un stade juvénile (18 à 20 j, 2 à 3 feuilles développées) par des préparations d'ARN viral purifié, dilué dans un tampon phosphate disodique $0,03 \mathrm{M}, \mathrm{pH} 8,3$, stérile, additionnées de carborandum (400 mesh, $75 \mathrm{mg} / \mathrm{ml}$ ).

Durant leur culture et leur incubation, les plantes sont maintenues dans un compartiment de serre « insect-proof», maintenu à $16-17^{\circ} \mathrm{C}$ durant la nuit et $23-24^{\circ} \mathrm{C}$ durant le jour, équipé d'un dispositif de lumière additionnelle afin d'assurer $16 \mathrm{~h}$ de jour en toute saison.

\section{B. Purification du virus et des ARN}

Toutes les souches sont multipliées chez la tomate et purifiées $15 \mathrm{j}$ après leur inoculation. La méthode de purification utilisée est celle décrite par LOT et al. (1972).

L'ARN viral est extrait des nucléoprotéines purifiées par 2 extractions successives au phénol dans le tampon Tris 0,02 $\mathrm{M}, \mathrm{NaCl} 0,2 \mathrm{M} ; \mathrm{pH} 7,0$, stérile, en présence de SDS 0,1 p. 100 et de bentonite 0,2 p. 100 . Après 2 précipitations par de l'éthanol froid, il est conservé à $-20^{\circ} \mathrm{C}$ dans de l'eau distillée.

L'ARN-5 est purifié par fractionnement de l'ARN total sur un gradient 5-25 p. 100 de saccharose pur dans le tampon Tris $0,01 \mathrm{M}, \mathrm{NaCl} 0,1 \mathrm{M} ; \mathrm{pH} \mathrm{7,0}$ stérile. De telles préparations d'ARN-5 ne sont pas infectieuses sur $V$. sinensis à la concentration de $20 \mu \mathrm{g} / \mathrm{ml}$.
Figure 1

Tomate saine (à gauche) ou infectée par la souche $R$ (à droite). Healthy tomato plant (on the left); $C M V-R$ infected tomato plant (on the right).

\section{Figure 2}

A gauche, tomate saine; à droite, tomate infectée par la souche $I_{17} F$, présentant les symptômes de mosaïque et filiformisme grave.

On the left, healthy tomato plant; on the right, tomato plant infected with $I_{J 7} F$ strain, showing mosaic and severe fern leaf symptoms.

Figure 3

A gauche, tomate saine; à droite, tomate atteinte de la nécrose létale caractéristique.

On the left, healthy tomato plant; on the right, tomato plant showing typical lethal necrosis.
Figure 4

Tomates infectées par la souche $I_{17} F$ seule (à droite) ou additionnée de l'ARN-5 de la souche $R$ (à gauche) et présentant respectivement des symptomes de mosaïque et filiformisme grave ou atténué.

Tomato plants infected with $I_{17} F$ strain alone (on the right) or with $R$ $R N A-5$ added (on the left) and respectively showing severe or attenuated fern leaf symptoms.

Figure 5

Analyse, par gel de polyacrylamide, des composants ARN des particules purifiées à partir de tomates infectées par les souches $I_{17} F$ (A) ou $I_{17} N(B)$.

Polyacrylamide gel electrophoresis of virus-RNA purified from tomato plants inoculated with strains $I_{17} F(A)$ or $I_{17} N(B)$. 

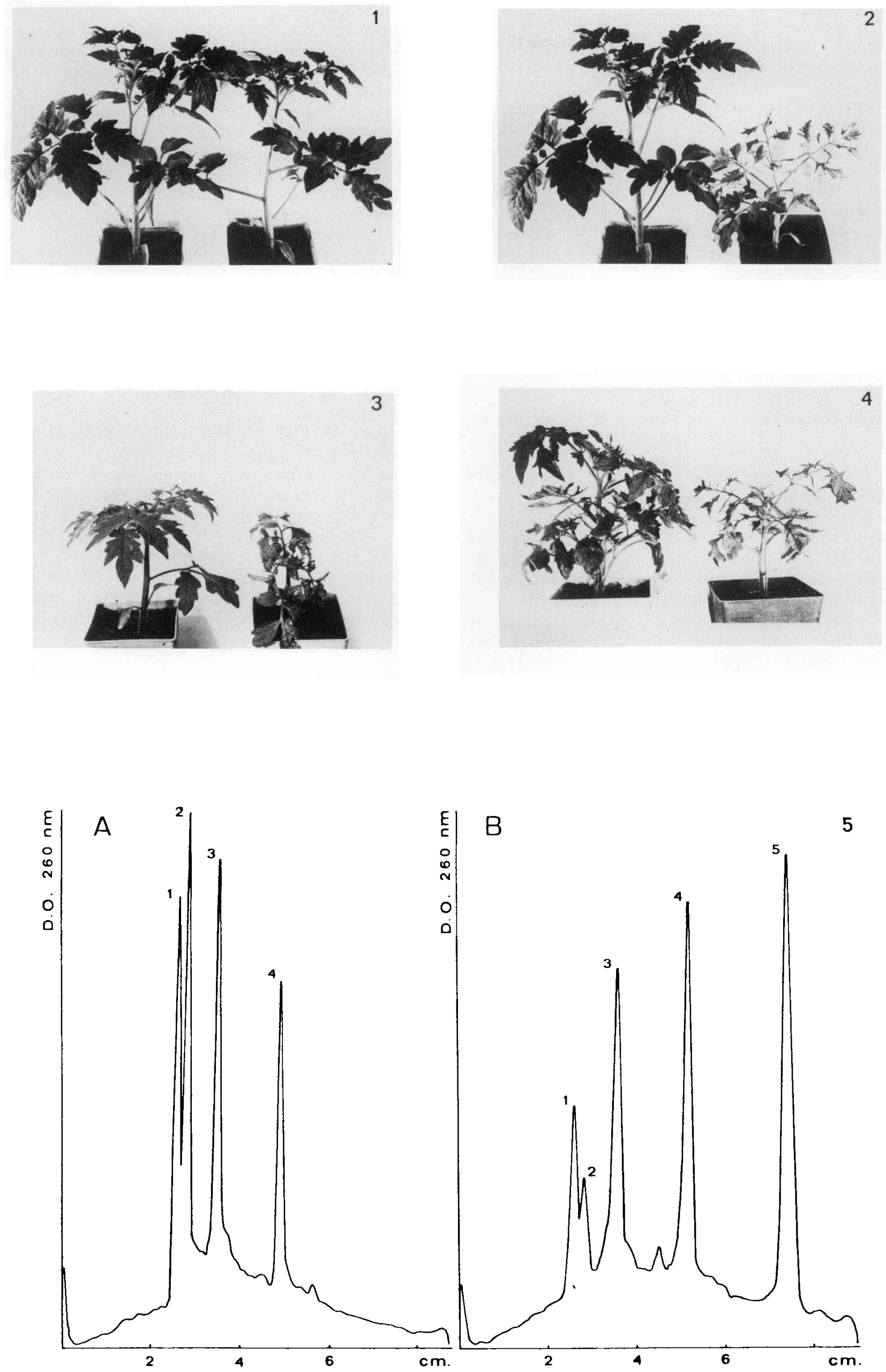


\section{Analyse en gel de polyacrylamide}

Des gels cylindriques $(0,6 \times 8,5 \mathrm{~cm})$ d'acrylamide $2,4 \mathrm{p}$. 100 sont préparés selon la méthode décrite par LOENING (1967) mais à pH 7,8 et en présence de SDS 0,1 p. 100.

$20 \mu \mathrm{g}$ d'ARN total ou $5 \mu \mathrm{g}$ d'ARN satellite sont déposés sur un gel après un chauffage à $60^{\circ} \mathrm{C}$ durant $3 \mathrm{mn}$ et un refroidissement rapide à $0^{\circ} \mathrm{C}$. La durée d'électrophorèse est de $3 \mathrm{~h}$ à raison de 4,3 $\mathrm{mA}$ par gel.

L'absorption des ARN à $260 \mathrm{~nm}$ est lue le long du gel à l'aide d'un spectrophotomètre GILFORD modèle 250.

La quantité relative de chaque composant est déterminée par la pesée de l'aire découpée correspondant au pic obtenu sur l'électrophorégramme.

\section{RÉSULTATS}

\section{A. Aptitudes pathogènes des souches de VMC chez la tomate}

La nature des symptômes observés chez la tomate après inoculation mécanique des diverses souches est présentée dans le tableau 2.

La souche $\mathrm{R}$ n'induit généralement aucun symptôme. On observe parfois une légère tendance à l'extériorisation de symptômes de filiformisme qui disparaissent par la suite. La longueur des entre-nœuds peut être réduite (fig. 1).

Les symptômes de filiformisme correspondent à la réduction de la surface de la feuille qui ressemble parfois à une lanière (souche $\mathrm{I}_{17} \mathrm{~F}$ en particulier). La taille des plantes est fortement réduite (fig. 2).

Le syndrome nécrotique se manifeste tout d'abord par l'enroulement vers le bas des feuilles apicales, perpendiculairement à leur nervure principale. Des lésions nécrotiques se développent sur les feuilles puis s'étendent en plages. Des stries brunes apparaissent le long des pétioles et rejoignent la tige. Ces nécroses, en s'étendant, ceinturent la tige et provoquent, dans les $15 \mathrm{j}$ qui suivent l'inoculation, le dessèchement total de la plante (fig. 3).

\section{B. Rôle de l'ARN-5 dans le développement de la nécrose létale chez la tomate}

L'analyse en gel de polyacrylamide du contenu nucléique des particules purifiées à partir de tomates infectées par les 8 souches de VMC met en évidence une corrélation entre la présence d'ARN-5 dans les virions et l'extériorisation du syndrome nécrotique (tabl. 2). Les symptômes de mosaïque et filiformisme sont associés par contre à des souches qui ne contiennent pas d'ARN satellite à l'exception de la souche R (tabl. 2).

La figure 5 représente les électrophorégrammes obtenus pour les ARN des souches $I_{17} F(A)$ et $I_{17} N(B)$. L'addition d'ARN-5 $(5 \mu \mathrm{g} / \mathrm{ml})$, extrait de l'ARN total de la souche $\mathrm{I}_{17} \mathrm{~N}$, à l'inoculum constitué par la préparation purifiée d'ARN de la souche $I_{17} F(20 \mu \mathrm{g} / \mathrm{ml})$, provoque le développement de la nécrose létale caractéristique chez la totalité des tomates ainsi contaminées.

De la même façon, l'adjonction des ARN satellites des souches $D$, TL ou $S$ aux ARN des souches $I_{17} F, K$ ou $M$ se traduit par l'extériorisation du syndrome nécrotique par les tomates inoculées.

\section{Incapacité pour l'ARN-5 de la souche $R$ à provoquer la nécrose}

L'apport d'ARN-5 purifié à partir de la souche $\mathrm{R}$, à la concentration de $5 \mu \mathrm{g} / \mathrm{ml}$, aux ARN de la souche $I_{17} F$ $(20 \mu \mathrm{g} / \mathrm{ml})$, n'induit pas de nécrose parmi les tomates infectées; il provoque au contraire chez celles-ci une importante réduction de la gravité des symptômes de filiformisme résultant de l'infection par le génome viral seul (fig. 4).

L'analyse du contenu nucléique des particules purifiées à partir de ces plantes montre que l'ARN-5 a été synthétisé puisqu'il représente 45 p. 100 environ de l'ARN encapsidé.

\section{Effet de l'addition simultanée de l'ARN-5 nécrogène et de l'ARN-5 de la souche $R$ dans l'inoculum}

L'existence d'un ARN satellite incapable de déclencher la nécrose létale lorsqu'il est associé au génome viral et l'atténuation des symptômes qu'il induit, nous ont amenés à étudier l'effet provoqué par l'addition des 2 types d'ARN satellite dans un inoculum inducteur de mosaique et filiformisme.

Le tableau 3 présente les résultats d'un des essais d'infection de tomates par des inoculums constitués des ARN de la souche $I_{17} F(20 \mu \mathrm{g} / \mathrm{ml})$ auxquels ont été ajoutées des doses croissantes des ARN-5 purifiés à partir des souches $\mathrm{R}$ et/ou $\mathrm{I}_{17} \mathrm{~N}$.

Quand les ARN-5 de la souche $I_{17} \mathrm{~N}$ ou de la souche $\mathrm{R}$ sont apportés indépendamment dans l'inoculum à une dose supérieure ou égale à $5 \times 10^{-3} \mu \mathrm{g} / \mathrm{ml}$, la totalité des toma-

TABLEAU 2

Symptômes induits chez la tomate par les 8 souches de VMC et proportion relative d'ARN-5 dans les nucléoprotéines purifiées Symptoms induced on tomato plants after infection with the $8 \mathrm{CMV}$ strains and relative proportion of RNA-5 in purified virus

\begin{tabular}{|c|c|c|}
\hline Souche & $\begin{array}{c}\text { Nature des symptômes développés } \\
\text { par les tomates }\end{array}$ & $\begin{array}{l}\text { p. } 100 \text { d'ARN-5 dans } \\
\text { les nucléoprotéines virales }\end{array}$ \\
\hline $\mathrm{I}_{17} \mathrm{~F}$ & Mosaïque et filiformisme grave & 0 \\
\hline $\mathrm{I}_{17} \mathrm{~N}$ & Nécrose létale & 45 à 50 \\
\hline D & Nécrose létale & 20 à 25 \\
\hline $\mathrm{TL}$ & Nécrose létale & 40 à 45 \\
\hline$M$ & Mosaïgue et filiformisme & 0 \\
\hline $\mathrm{S}$ & Nécrose létale & 30 à 35 \\
\hline $\mathrm{K}$ & Mosaïque et filiformisme faible & 0 \\
\hline $\mathrm{R}$ & Filiformisme très faible ou pas de symptome & 40 à 45 \\
\hline
\end{tabular}




\section{TABLEAU 3}

Proportion des différents types de symptômes observés sur tomate après inoculation de la souche $I_{17} F$ en présence des $A R N-5 I_{17} N$ et $R$ seuls ou en mélange

Symptoms induced on tomato plants after inoculation with $C M V$ $I_{17} F R N A$ and $R N A-5$ of strains $I_{17} N$ and $R$ added alone or together

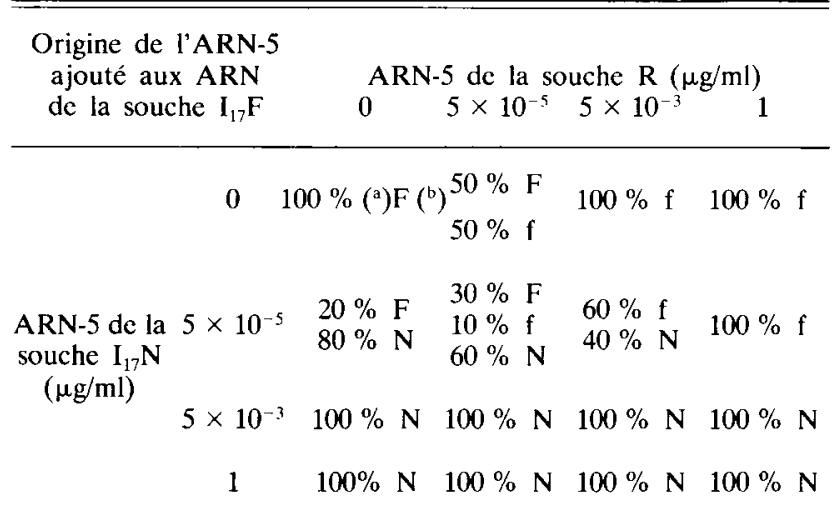

(a) Les pourcentages sont calculćs à partir de 10 tomates inoculces. (Each value is given for 10 inoculated plants).

(b) F : mosaïque et filiformisme grave (mosaic and severe fern leaf symptoms)

$\mathrm{f}$ : mosaïque et filiformisme atténué (mosaic and attenuated fern leaf symptoms)

$\mathrm{N}$ : nécrose létale (lethal necrosis).

tes extériorisent respectivement la nécrose ou des symptômes de filiformisme atténué. Cette concentration paraît suffisante pour que, dans toutes les plantes inoculées, l'ARN satellite soit activement multiplié. A une dose inférieure, cette synthèse devient aléatoire puisqu'un certain pourcentage de tomates présente le symptôme de filiformisme grave, conséquence de la multiplication du seul génome viral.

Ces résultats confirment ceux obtenus au cours de l'étude de la dose minimale requise pour assurer la synthèse du satellite (JACQUEMOND \& LEROUX, 1982).

Lorsqu'ils sont simultanément apportés dans l'inoculum, la totalité des tomates se nécrosent si l'ARN satellite $I_{17} N$ est présent à une dose égale ou supérieure à $5 \times 10^{-3} \mu \mathrm{g} / \mathrm{ml}$, et cela, quelle que soit la concentration de l'ARN satellite $R$. On n'observe alors aucun effet protecteur de ce dernier, même s'il se trouve à une dose 200 fois supérieure à celle de l'ARN-5 nécrogène.

Quand l'ARN satellite $\mathrm{I}_{17} \mathrm{~N}$ est additionné à la dose de $5 \times 10^{-5} \mu \mathrm{g} / \mathrm{ml}$, les tomates n'extériorisent plus du tout le syndrome nécrotique si l'ARN satellite $\mathrm{R}$ est présent à une concentration égale ou supérieure à $5 \times 10^{-3} \mu \mathrm{g} / \mathrm{ml}$, ou moins fréquemment s'il se trouve en quantité plus faible.

Ces résultats illustrent vraisemblablement la compétition de synthèse entre les 2 ARN satellites.

\section{DISCUSSION}

Nos résultats confirment après KAPER \& WATERWORTH (1977), JACQUEMOND (1980) et plus récemment TAKANAMI (1981), la responsabilité directe de l'ARN satellite associé au VMC dans le développement de la nécrose létale de la tomate. Ils démontrent que ce syndrome caractéristique peut être induit par plusieurs isolats naturels de VMC contenant l'ARN satellite comme par des inoculums constitués par les ARN génomiques et l'ARN satellite isolé de souches homologues ou hétérologues. Les ARN-5 de nos souches ont, par ailleurs, des propriétés très voisines de celles décrites par les autres auteurs pour leurs isolats (JACOUEMOND \& LEROUX, 1982).

L'ARN satellite (SAT-RNA) étudié par l'équipe australienne (GOULD et al., 1978; MOSSOP \& FRANCKI, 1978, 1979) possède de nombreuses propriétés communes avec les ARN-5 étudiés par l'équipe américaine ou par nous-mêmes. Il diffère de ceux-ci (ARN-5 de la souche R excepté) par son inaptitude à induire le syndrome nécrotique létal de la tomate.

FRANCKI, et al. (1980), ayant isolé de tomates présentant au champ des symptômes de nćcrose un complexe de VMC et du virus de la mosaïue de la luzerne ( $A M V$, alfalfa mosaic virus) et établi la responsabilité de ce dernicr dans le développement de ces symptômes, font l'hypothèse que les isolats des autres équipes sont peut-être contaminés par l'AMV. Plusieurs virus ou complexes de virus sont en effet capables de provoquer des symptômes de nécrose chez la tomate (MARrou et al., 1974) et, parmi eux, l'AMV (MARROU \& Migliori, 1966). Les différents contrôles auxquels nous avons soumis nos isolats permettent d'affirmer, après MARROU \& DUTEIL (1974), que nos souches de VMC ne sont pas contaminées et peuvent, en tant que telles, induire le syndrome nécrotique caractéristique de la tomate.

La différence d'aptitude pathogène du SAT-RNA et de l'ARN-5 de la souche $S$ étudié par KAPER \& WATERWORTH (1977) paraît plus vraisemblablement liée aux différences de séquences nucléotidiques observées entre ces molécules par MOSSOP \& FRANCKI (1979).

Les résultats que nous avons obtenus avec la souche $R$ confirment qu'il peut effectivement exister des « souches" d'ARN satellite incapables d'induire le syndrome nécrotique de la tomate lorsqu'elles sont associées au VMC. Une précédente étude avait suggéré que les variations de séquence entre les $A R N-5$ des souches $D, T L, I_{17} N$ ou $S$ sont très réduites (RICHARDS et al., 1978). Les résultats de l'étude comparée des séquences de l'ARN-5 de la souche $R$ et des autres ARN-5 n'apportent pas la preuve d'importantes différences de structure primaire de ces molécules (JACQUEMOND, RICHARDS \& JONARD, résultats non publiés) et confirment les résultats antérieurs de KAPER \& Tousignant (1978) qui disposent également de la souche $\mathbf{R}$.

La comparaison systématique des séquences nucléotidiques et des éventuels produits de traduction d'ARN satellites ayant ou non des aptitudes nécrogènes devrait être entreprise. Compte tenu des résultats actuels, obtenus par plusieurs équipes ayant étudié les homologies de séquence à l'aide de méthodes très différentes, il semblerait qu'il existe 3 types d'ARN satellite:

- ARN-5 nécrogène du type de l'ARN-5 de la souche D dont la séquence a été déterminée dans sa totalité (RICHARDS et al., 1978);

- ARN-5 non nécrogène, de type $R$, dont la séquence est très voisine de celle du précédent;

- SAT-RNA, non nécrogène, présentant seulement 70 p. 100 d'homologie de séquence avec les précédents.

L'ensemble de ces résultats constitue un élément intéressant pour la compréhension de l'étiologie et de l'épidémiologie de la maladie - sous ses différents faciès - provo- 
quée par le VMC en culture de tomates. Il est maintenant établi que le développement de la nécrose est provoqué, dans de nombreux cas, par le VMC, et cela, quand la souche possède un ARN satellite de type $D$. De très nombreux isolats réalisés à partir de plusieurs espèces hôtes ont été identifiés comme étant du VMC porteur d'ARN satellite et inducteur de nécrose sur tomate. Par contre, le symptôme de filiformisme à des degrés divers peut résulter de l'infection par un isolat dépourvu d'ARN satellite ou porteur d'ARN-5 de type R. Ce dernier type d'isolat peut aussi ne provoquer aucun symptôme sur la plante qu'il infeste. QUIOT et al. (1979) avaient noté que certaines tomates, apparemment saines, étaient effectivement contaminées, depuis plusieurs semaines par le VMC. La caractérisation des souches - et des ARN-5 qui leur sont associés pouvant infecter seules ou en mélange les tomates au champ est à poursuivre à la lumière de ces nouvelles acquisitions.

La réduction importante de la gravité des symptômes sur tomate, résultant de l'association d'ARN-5 de la souche $R$ au VMC, constitue un nouvel exemple de modification de symptômes liée à la présence de l'ARN satellite (KAPER \& WATERWORTH, 1977; MOSSOP \& FRANCKI, 1978; WATERWORTH et al., 1979 ; JACQUEMOND, 1980 ; TAKANAMI, 1981). L'étude de la protection conférée par une inoculation de souches peu pathogènes sur tomate et pourvues de l'ARN-5 de type $\mathrm{R}$ vis-à-vis d'isolats nécrogènes, basée sur les interférences entre les 2 types d'ARN satellite, est actuellement entreprise.

Reçu le 16 avril 1981 Accepté le 26 août 1981.

\section{REMERCIEMENTS}

Les photographies de cet article sont dues à Jacques Rougier que nous remercions.

\section{RÉFÉRENCES BIBLIOGRAPHIQUES}

Devergne J. C., Cardin L., Marais A., 1969. Etude d'une souche du virus de la mosaïque du concombre isolée en France de Ranunculus asiaticus L. Ann. Phytopathol., 1, H.S., 329-337.

Diaz-Ruiz J. R., Kaper J. M., 1977. Cucumber mosaic virusassociated RNA-5. III. Little nucleotide sequence homology between CARNA-5 and helper RNA. Virology, 80, 204-213.

Francki R. I. B., Gould A. R., Hatta T., 1980. Variation in the pathogenicity of three viruses of tomato. Ann. appl. Biol., 96, 219226.

Gould A. R., Palukaitis P., Symons R. H., Mossop D. W., 1978. Characterization of a satellite RNA associated with cucumber mosaic virus. Virology, 84, 443-445.

Jacquemond M., 1980. Propriétés et fonctions biologiques du satellite du virus de la mosaique du concombre. La nécrose de la tomate. Thèse de docteur-ingénieur. Université d'Aix-Marseille, $167 \mathrm{p}$.

Jacquemond M., Leroux J. P., 1982. L'ARN satellite du virus de la mosaïque du concombre. II. Etude de la relation virus-ARN satellite chez divers hôtes. Agronomie, 2 (1) (à paraître).

Kaper J. M., Tousignant M. E., 1977. Cucumber mosaic virus associated RNA-5. I. Role of host plants and helper strains in determining amount associated with virions. Virology, 80, 186-195.

Kaper J. M., Tousignant M. E., 1978. Cucumber mosaic virusassociated RNA-5. V. Extensive nucleotide sequence homology among CARNA-5 preparations of different CMV strains. Virology, 85, 323-327.

Kaper J. M., Tousignant M. E., Lot H., 1976. A low molecular weight replicating RNA associated with a divided genome plant virus : defective or satellite RNA? Biochem. biophys. Res Commun., 72 (4), 1237-1243.

Kaper J. M., Waterworth H. E., 1977. Cucumber mosaic virusassociated RNA-5 causal agent for tomato necrosis. Science, 196, 429-431.

Loening U. E., 1967. The fractionation of high molecular weigh ribonucleic acid by polyacrylamide gel electrophoresis. Biochem. J., 102, 251-257.

Lot H., Kaper J. M., 1976. Further studies on the RNA component distribution among the nucleoproteins of cucumber mosaic virus. Virology, 74, 223-226.

Lot H., Jonard G., Richards K. E., 1977. Cucumber mosaic virus RNA-5. Partial characterization and evidence for no large sequence homologies with genomic RNAs. Febs Letters, 80 (2), 395-399.

Lot H., Marchoux G., Marrou J., Kaper J. M., West C. K., Van Vloten Doting L., Hull R., 1974. Evidence for three functional RNA species in several strains of cucumber mosaic virus. J. Gen. Virol., 22, 81-93.
Lot H., Marrou J., Quiot J. B., Esvan C., 1972. Contribution à l'étude du virus de la mosaique du concombre (CMV). I. Méthode de purification rapide du virus. Ann. Phytopathol., 4 (1), 25-38.

Marchoux G., 1975. Propriétés biologiques et génétiques des ARN $d u$ virus de la mosaique du concombre. Thèse de docteur ès sciences naturelles. Université d'Aix-Marseille. 134 p.

Marrou J., Duteil M., 1974. La nécrose de la tomate. Reproduction des symptômes de la maladie par inoculation mécanique de plusieurs souches du virus de la mosaique du concombre (VMC). Ann. Phytopathol., 6 (2), 155-172.

Marrou J., Duteil M., Lot H., Clerjeau M., 1973. La nécrose de la tomate. Une grave virose des tomates cultivées en plein champ. Pépiniéristes Hortic. Maraichers, 137, 37-41.

Marrou J., Duteil M., Schmidt R., 1974. Identification de plusieurs virus capables de provoquer un syndrome nécrotique sur tomate. Ann. Phytopathol. 6, 228.

Marrou J., Migliori A., 1966. Réaction de la tomate au virus de la mosaïque de la luzerne. Etudes de Virologie, Ann. Epiphyt., 17, H.S., 113-120.

Mossop D. W., Francki R. I. B., 1978. Survival of a satellite RNA in vivo and its dependance on cucumber mosaic virus for replication. Virology, 86, 562-566.

Mossop D. W., Francki R. I. B., 1979. Comparative studies on two satellites RNAs of cucumber mosaic virus. Virology, 95, 395-404. Peden K. W. C., Symons R. H., 1973. Cucumber mosaic virus contains a functionally divided genome. Virology, 53, 487-492.

Putz C., Kuszala J., Kuszala M., Spindler C., 1974. Variation du pouvoir pathogène des isolats du virus de la mosaïque du concombre associée à la nécrose de la tomate. Ann. Phytopathol., 6 (2), 139-154.

Quiot J. B., Douine L., Gebre Selassie K., 1979. Fréquence des principales viroses identifiées dans une exploitation maraîchère du Sud-Est de la France. Ann. Phytopathol., 11 (3), 283-290.

Richards K. E., Jonard G., Jacquemond M., Lot H., 1978. Nucleotide sequence of cucumber mosaic virus - associated RNA5. Virology, 89, 395-408.

Takanami Y., 1981. A striking change in symptoms on cucumber mosaic virus - infected tobacco plants induced by a satellite RNA. Virology, 109, 120-126.

Takanami Y., Kubo S., Imaizumi S., 1977. Synthesis of single and double stranded cucumber mosaic virus RNAs in tobacco mesophyll protoplasts. Virology, 80, 376-389.

Waterworth H. E., Kaper J. M., Tousignant M. E., 1979. CARNA 5 , the small cucumber mosaic virus - dependent replicating RNA, regulates disease expression. Science, 204, 845-847. 